\title{
Elastoplastic Model of Sand under Complex Loading
}

\author{
Z. Wan, ${ }^{\mathrm{a}, 1}$ C. C. Song, ${ }^{\mathrm{b}}$ and W. S. Gao ${ }^{\mathrm{a}}$ \\ ${ }^{a}$ China Academy of Building Research, Research Institute of Base and Foundation, Beijing, China \\ ${ }^{\mathrm{b}}$ Institute of Structural Engineering and Disaster Reduction, Tongji University, Shanghai, China \\ 1'zhengw111@126.com
}

The unified hardening model for overconsolidated clay is extended to the cyclic model considering the stress-strain relationship for sands by adopting the rotational hardening rule. The equations for the normal consolidated line and critical state line are revised in the e-lnp space to describe the breakage behavior of gravel sands under high pressures. The power function is used to define the above equations. The model features the following characteristics: (1) equations for the above lines are redefined in the e-lnp space; (2) potential strength is revised by the Hvorslev line containing the power parameter; (3) cyclic behavior of sands is described by the evolutionary rule of current yield surface and rotational hardening rule; (4) generalized cyclic model is realized using the transformed stress method based on the spatially mobilized plane criterion. Simulation results show that the breakage and cyclic loading behavior of gravel sands are adequately described.

Keywords: sands, rotational hardening, transformed stress method, cyclic loading.

Introduction. A lot of stress path tests about clay showed that there is a specific mapping relationship between the mean stress and void ratio. The normal compression line (NCL) of normally consolidated clay comes into a regular straight line in the $e-\ln p$ space. Meanwhile, the critical state enters into a straight line parallel with it, which reflects the unique correspondence characteristics of the mean stress and void ratio. It is different from clay for sand which does not have the unique correspondence characteristics of the mean stress and void ratio. Due to the different particle arrangement of sand, the same void ratio can correspond to different normal consolidation pressure. As Fig. 1 shows, in a broader range of pressure, the normal compression line in $e-\ln p$ space is no longer a straight line, and the critical state line (CSL) of sand is no longer a regular straight line. In the low-stress range, the slope is larger than that of the normal compression. Similar to the sand, coarse-grained soil also belongs to the cohesionless soil, which has the same characteristics with the sand. However, due to large confining pressure, particle breakage occurs between coarse-grained soils. This results in the filling of geotechnical materials broken into the large void, meanwhile the void ratio will be smaller. As compared to the unbroken coarse-grained soil, the volume change is more significant. With the confining pressure gradually increased, the amount of compression volume rises. When the confining pressure reaches a high value, the increase in the volume compression becomes slight. Different soil tests by Verdugo and Ishihara [1], Yamamuro and Lade [2] have already observed this phenomenon, Muir Wood [3] carried out some research on the above characteristics.

To describe the deformation characteristics of sand more accurately and overcome the defects of single logarithmic coordinate, Hashiguchi [4] takes the double logarithmic coordinate form in $e-\ln p$ space, describing the clay and sand are in a unified mathematical form. Using the double logarithmic coordinates, the relationship between volume change and mean stress of sand can be considered in the broad range. Also, under the larger mean stress, the issue which void ratio cannot be negative is naturally taking into account. Because the void ratio is a sensitive parameter for the sand, using logarithmic coordinates is equivalent to the amplification of the void ratio unit. Therefore, when $\Delta \ln p$ changes in 


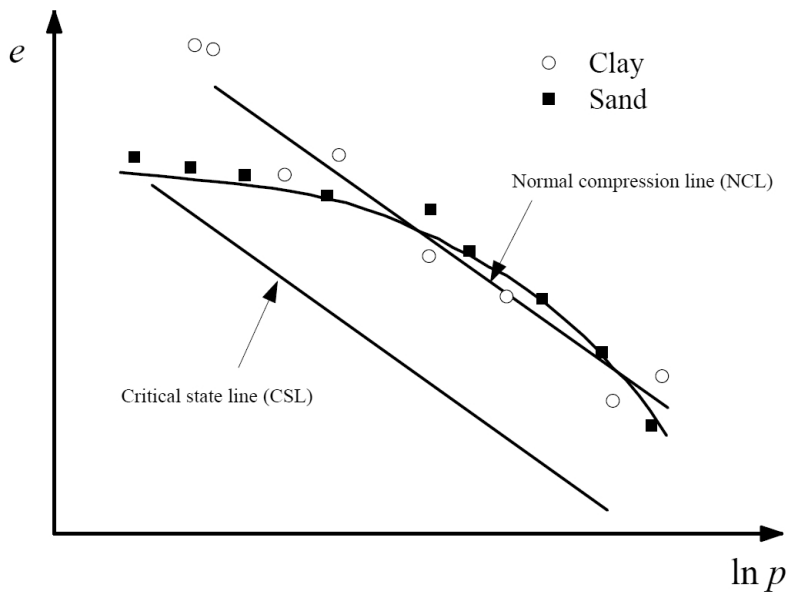

Fig. 1. Normal compression line of sand and clay in $e-\ln p$ space.

the small range, it will lead to the more significant change of void ratio and larger variable description. Nakai [5] takes the form of power function to express the relationship between the curve in the $e-\ln p$ space. Gudehus [6] and others simulated the relationship between the void ratio and the mean stress in $e-\ln p$ space by a nonlinear function, which can be well simulated in a wide range of mean stresses.

Different from clay, the NCL corresponds to the relationship between the initial mean stress and void ratio, while the sand has no strict normal compression line as clay. The initial compression line and void ratio are not unique, so it is meaningless to measure the compaction characteristics of sand with over consolidation degree. Ishihara and Yasuda [7] and Zhang et al. [8] carried out various tests on the stress path of sand. The conclusion was also confirmed that the deformation characteristics of sand are very little related to the previous stress history, or the influence of stress path on its deformation can be neglected, and the current state of stress is affected by its deformation. Li and Wang [9] proposed the state dependent theory to describe the characteristics of sand. The present state of compaction is represented by the distance between the void ratio of the current mean stress and the critical void ratio. The shear and dilatancy characteristics of sand can be simulated better by using the state parameter $\psi$. However, the above model applies to static loading conditions, the simulation under complex loading conditions is still rare.

1. Normal Compression Line and the Critical State Line. Considering that in the $e-\ln p$ space, the non-dimensional power function of standard atmospheric pressure is using as abscissa and void ratio is using as ordinate, while the corresponding NCL exists in the space. Due to the confining pressure dependence of sand, when the confining pressure is zero, i.e., the mean stress is zero, the normal compression line should coincide with the void ratio of the critical state line. But the slope of the normal compression line is lower, while the slope of the critical state line is higher. There is a reference line (RL) below the NCL, and the RL is obtained according to the CSL of the clay. According to the MCC model, under the constant $P$ path in the $e-\ln p$ space, the plastic strain which is from the normal consolidation line to the CSL should be $\ln 2(\lambda-\kappa) /\left(1+e_{0}\right)$. Similarly, as Fig. 2 shown under the constant $P$ path in the $e-\left(p / p_{a}\right)^{m}$ space, the plastic strain which is from the NCL to the RL can be determined as a definite value.

It is shown from Fig. 2 that the RL intersects the CSL at an intersection point $p_{\text {con }}$. When $p<p_{c o n}$, the volume change is not larger than $\left(c_{t}-c_{e}\right) \ln 2$. When $p>p_{c o n}$, the volume change is greater than $\left(c_{t}-c_{e}\right) \ln 2$. With the increase of the mean stress, the volume strain increases, too. It can reflect the phenomenon that the volume strain increases 


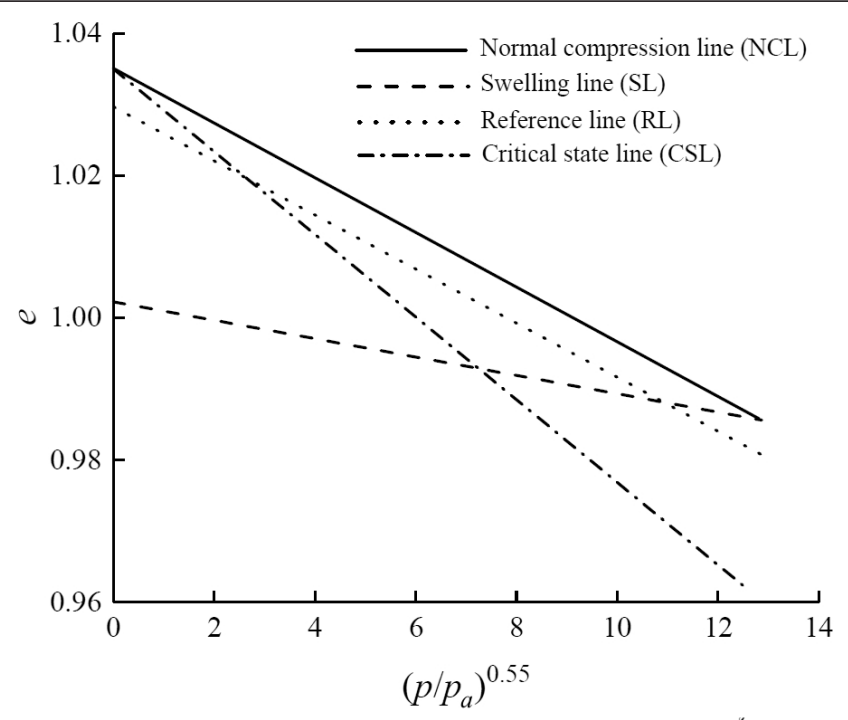

Fig. 2. Normal consolidated line and critical state line in $e-\left(p / p_{a}\right)^{\xi}$ space.

with the rise of confining pressure, while in the low-stress range, the volume becomes smaller, which indicates the change of volume strain with the shift in the mean stress. According to the experimental phenomena under certain pressure range, the normal compression line, swelling line, reference line and critical state line of the sand can be expressed as straight lines in the $e-\left(p / p_{a}\right)^{m}$ space as follows.

Normal compression line equation (NCL):

$$
\text { NCL: } e_{N}=e_{N 0}-c_{t}\left(\frac{p}{p_{a}}\right)^{m} .
$$

Swelling line equation (SL):

$$
\mathrm{SL}: e_{s}=e_{s 0}-c_{e}\left(\frac{p}{p_{a}}\right)^{m} .
$$

Reference line equation (RL):

$$
\mathrm{RL}: e_{c}=e_{c 0}-c_{t}\left(\frac{p}{p_{a}}\right)^{m} .
$$

Critical state line equation (CSL):

$$
\text { CSL: } e_{c s}=e_{N 0}-\alpha_{c}\left(\frac{p}{p_{a}}\right)^{m} \text {. }
$$

The reference point is the interception of the reference line and critical state line: $\left(e_{c o n}, p_{c o n}\right)$. The interception of a reference line with void ratio can be deduced as $\left(e_{c 0}, 0\right) \Rightarrow\left(\left(1+e_{N 0}\right)\left(1-\varepsilon_{v c}^{p}\right)-1,0\right)$. 
Thereinto,

$$
\varepsilon_{v c}^{p}=\left(c_{t}-c_{e}\right) \ln 2
$$

The interception is

$$
\left(\frac{p_{c o n}}{p_{a}}\right)^{m}=\frac{\left(e_{c 0}-e_{N 0}\right)}{\left(c_{t}-\alpha_{c}\right)}
$$

Then, under any mean stress, the plastic strain produced by the loading of the constant $P$ path from NCL to CSL line can be expressed as

$$
\varepsilon_{v}^{p}=\varepsilon_{v c}^{p} \frac{\left(p / p_{a}\right)^{m}}{\left(p_{c o n} / p_{a}\right)^{m}}=\left(c_{t}-c_{e}\right) \ln 2 \frac{\left(c_{t}-\alpha_{c}\right)}{\left(e_{c 0}-e_{N 0}\right)}\left(p / p_{a}\right)^{m}=c_{p 2}\left(p / p_{a}\right)^{m} .
$$

Then $p_{0} \rightarrow p$,

$$
\varepsilon_{v}^{p}=c_{p 2}\left[\left(\frac{p}{p_{a}}\right)^{m}-\left(\frac{p_{0}}{p_{a}}\right)^{m}\right]
$$

\section{Reference Yield Surface and Current Yield Surface.}

2.1. Reference Yield Surface. The unified hardening cyclic model is transformed into three dimensions by using the transformation stress method [10]. The reference yield surface and the lower loading surface in the transformed stress space are obtained. The reference yield surface equation can be expressed as

$$
\widetilde{f}_{r}=\frac{c_{p}}{p_{a}^{m}}\left\{\left[\overline{\widetilde{p}}\left(1+\frac{\tilde{\eta}^{* 2}}{M^{2}-\tilde{\zeta}^{2}}\right)\right]^{m}-\left(\overline{\widetilde{p}}_{0}\right)^{m}\right\}-\varepsilon_{v}^{p}=0 .
$$

Here, $c_{p}=c_{t}-c_{e}, c_{t}$ is the slope of the normal compression line, $c_{e}$ is the slope of the swelling line, $p_{a}=98 \mathrm{kPa}, \overline{\widetilde{p}}_{0}$ is the maximum compression mean stress for history, $\widetilde{\zeta}$ is the axis of the yield surface,

$$
\widetilde{\zeta}=\sqrt{\frac{3}{2} \widetilde{\beta}_{i j} \widetilde{\beta}_{i j}}
$$

$\widetilde{\eta}^{*}$ is the relative stress ratio,

$$
\widetilde{\eta}^{*}=\sqrt{\frac{3}{2} \widetilde{\widetilde{\eta}}_{i j} \widehat{\widetilde{\eta}}_{i j}}=\sqrt{\frac{3}{2}\left(\widetilde{\eta}_{i j}-\widetilde{\beta}_{i j}\right)\left(\widetilde{\eta}_{i j}-\widetilde{\beta}_{i j}\right)}
$$

and $\tilde{\eta}_{i j}$ is the stress ratio of component,

$$
\tilde{\eta}_{i j}=\tilde{s}_{i j} / \tilde{p}=\left(\widetilde{\sigma}_{i j}-\tilde{p} \delta_{i j}\right) / \tilde{p}
$$

The axis component is obtained by the incremental method, and the increment formula is as follows:

$$
\widetilde{d}_{i j}=\frac{b_{r} M}{c_{p 2}}\left(m_{b} M-\tilde{\zeta}\right) d \varepsilon_{d}^{p} \frac{\hat{\tilde{\eta}}_{i j}}{\|\hat{\tilde{\eta}}\|}
$$


2.2. Current Yield Surface. According to the associated flow rule, the potential plastic surface and under loading surface are the same. The under loading surface equation is

$$
\widetilde{f}=\frac{c_{p}}{p_{a}^{m}}\left\{\left[\tilde{p}\left(1+\frac{\tilde{\eta}^{* 2}}{M^{2}-\tilde{\zeta}^{2}}\right)\right]^{m}-\left(\tilde{p}_{0}\right)^{m}\right\}-H=0 .
$$

Unified hardening parameter:

$$
H=\int\left[\frac{M_{f}^{4}-\widetilde{\eta}^{4}}{M^{4}-\tilde{\eta}^{4}} d \varepsilon_{v}^{p}+A d \varepsilon_{d}^{p}\right]
$$

Potential strength parameter:

$$
M_{f}=6(\sqrt{k(1+k)}-k)
$$

where $k=\frac{M^{2}}{12(3-M) R^{\beta}}, \beta$ is the parameter.

Over-compression stress ratio parameter $R$ :

$$
\begin{gathered}
R=\tilde{p}\left[1+\overline{\widetilde{\eta}}^{* 2} /\left(M^{2}-\widetilde{\zeta}^{2}\right)\right]\left[\left(\overline{\tilde{p}}_{0}\right)^{m}+\left(p_{a}\right)^{m} \varepsilon_{v}^{p} / c_{p 2}\right]^{-1 / m} \\
A=\sqrt{\frac{3}{2}} b_{r} \widetilde{\eta}\left(m_{b} M-\widetilde{\zeta}\right) \frac{m}{p_{a}^{m}}\left[\widetilde{p}\left(1+\frac{\widetilde{\eta}^{* 2}}{\left(M^{2}-\widetilde{\zeta}^{2}\right)}\right)\right]^{m-1}\left(\frac{\widetilde{p}^{*}\left(3 \widetilde{\eta}_{i j} \widetilde{\beta}_{i j}-2 M^{2}\right)}{\left(M^{2}-\widetilde{\zeta}^{2}\right)^{2}}\right) .
\end{gathered}
$$

\section{Test Verification.}

3.1. Static Loading Simulation. To test the deformation characteristics under high confining pressure, the sand with confining pressure from 200 to $8000 \mathrm{kPa}$ was simulated [11]. The material parameters are shown in Table 1.

$\mathrm{T}$ a b 1 e 1

Material Parameters for Toyoura Sand

\begin{tabular}{|c|c|c|c|c|c|c|c|c||}
\hline$M$ & $c_{t}$ & $c_{e}$ & $v$ & $b_{r}$ & $m_{b}$ & $\alpha$ & $m$ & $\alpha_{c}$ \\
\hline 1.35 & 0.0044 & 0.0016 & 0.3 & 1.5 & 0.4 & 0.3 & 0.5 & 0.0158 \\
\hline
\end{tabular}

Figure 3a is a relationship curve between axial strain and the void ratio under the conventional triaxial compression condition. The overall strength of prediction value is larger than test value; the stress value is different under different confining pressure when the confining pressure is 0.2 or $0.5 \mathrm{MPa}$, the strength value is larger than the experimental value, while the confining pressure is $1.0 \mathrm{MPa}$, the strength value is smaller than the experimental value. For the confining pressure values of 2.0 and $4.0 \mathrm{MPa}$, the strength values are in good agreement with the experimental value. When the confining pressure is 8.0 $\mathrm{MPa}$, the strength value is too much higher. It is likely to be due to the high confining pressure. The sand particles are broken, resulting in the internal friction angle decreases and the decrease of strength. With the increase of axial strain, the stress ratio tends to be 


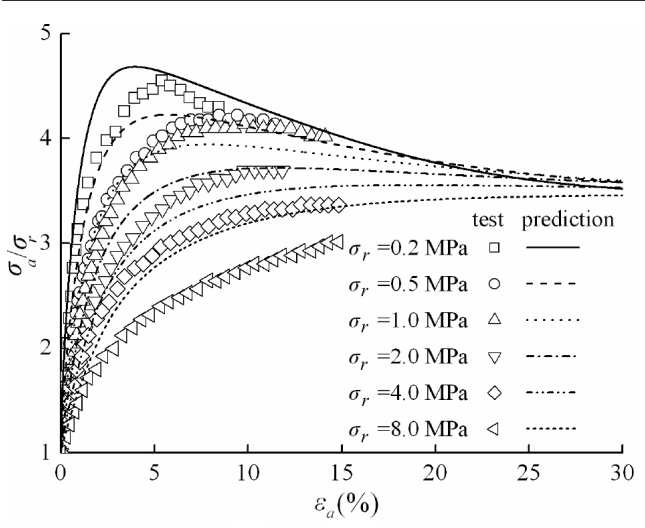

a

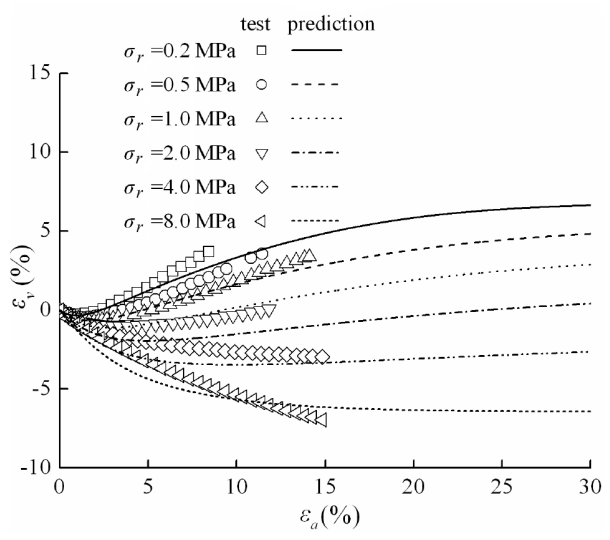

$\mathrm{b}$

Fig. 3. Test and simulated results of conventional triaxial compression on Toyoura sands.

consistent with the critical stress ratio, and it is close to the critical state. Figure $3 b$ is the relation between axial strain and volume strain corresponding to Fig. 3a. It can be seen that when the confining pressure is $0.2 \mathrm{MPa}$, the volume change value is in good agreement with the experimental data, and when the confining pressure is $0.5,1.0$, and $2.0 \mathrm{MPa}$, volume dilatancy rate is less than the test value, when the confining pressure is 4.0 and 8.0 $\mathrm{MPa}$, the volume change value is agreement with experimental values.

3.2. Cyclic Loading Simulation. To test the model under cyclic loading, different sand paths are chosen to simulate various stress paths.

Figure 4 is the conventional triaxial drained shear simulation for the selected Hostun dense sand [12], and the material parameters are shown in Table 2. The initial void ratio is 0.61 , the initial consolidation pressure is $350.0 \mathrm{kPa}$, the NCL intercept $N=1.037$, and the initial over consolidation stress ratio is $R=0.1$.

T a b 1 e 2

Material Parameters for Hostun Sand

\begin{tabular}{||c|c|c|c|c|c|c|c|c||}
\hline \hline$M$ & $c_{t}$ & $c_{e}$ & $v$ & $b_{r}$ & $m_{b}$ & $\alpha$ & $m$ & $\alpha_{c}$ \\
\hline 1.36 & 0.0044 & 0.0016 & 0.3 & 1.5 & 0.6 & 0.3 & 0.55 & 0.0158 \\
\hline
\end{tabular}

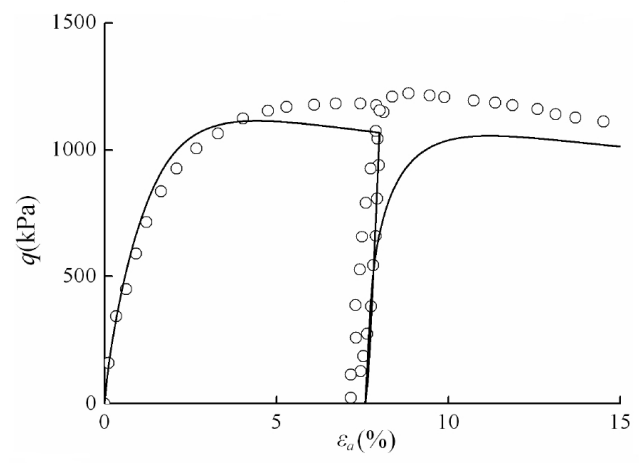

a

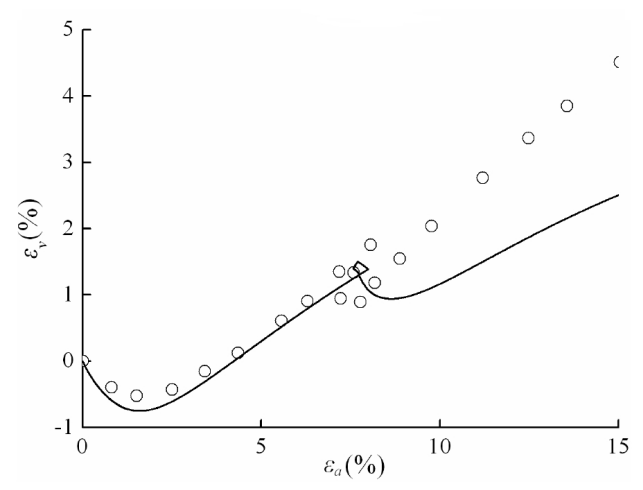

b

Fig. 4. Test and simulated results of cyclic triaxial compression on Hostun sands. 
The first cycle in Fig. $4 \mathrm{a}$ is in good agreement with the experimental value, and the hysteresis loop formed by the reloading curve is smaller than the experimental value, while the peak strength is smaller than the experimental value. Figure $4 \mathrm{~b}$ is the relationship curve between the axial strain and the volume strain. The first cycle agrees well with the experimental data, but the amplitude of dilatancy is smaller than the experimental value at the second cycle.

Figure 5 is a cyclic loading test of undrained stress path for Niigata loose sand made by Ishihara et al. [13]. The initial void ratio is 0.737 , the initial spherical stress is $212.6 \mathrm{kPa}$, the normal compression line intercept is $N=0.87$, while the initial over consolidation stress ratio is $R=1.0$. The material parameters are shown in Table 3.

T a b 1 e 3

\section{Material Parameters for Niigata Sand}

\begin{tabular}{|c|c|c|c|c|c|c|c|c||}
\hline$M$ & $c_{t}$ & $c_{e}$ & $v$ & $b_{r}$ & $m_{b}$ & $\alpha$ & $m$ & $\alpha_{c}$ \\
\hline 1.48 & 0.0070 & 0.0040 & 0.3 & 1.5 & 0.98 & 0.05 & 0.9 & 0.0188 \\
\hline
\end{tabular}

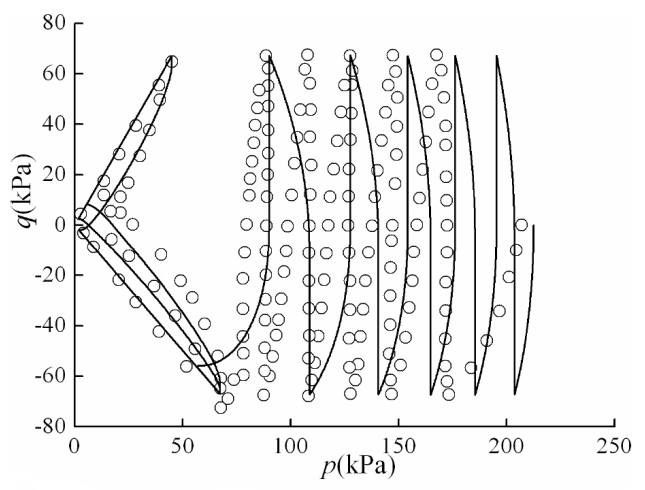

a

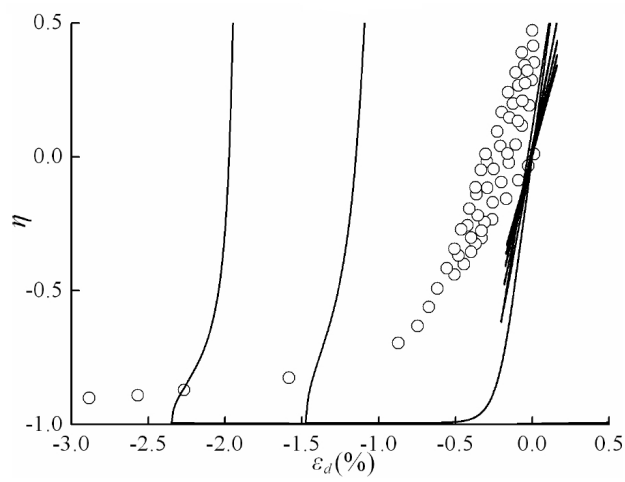

b

Fig. 5. Test and simulated results of cyclic mobility under the undrained condition on Niigata sands.

Figure 5a is the simulation of undrained stress path; it can be seen that after the fifth loading cycle, the stress state began to enter the cyclic mobility phase when the stress state point was close enough to the original point, the stress state started a cyclic activity. Figure $5 \mathrm{~b}$ is the relationship curve between the corresponding deviatoric strain and stress ratio. It can be seen that the deviatoric strain is increased sharply when the mean stress reaches about $60 \mathrm{kPa}$ from $212.6 \mathrm{kPa}$ when entered into the stage of the cyclic mobility, the deviatoric strain is not stable. At this stage, the trend of deviatoric strain with the stress ratio is consistent with the experimental value.

Figure 6 is the constant $P$ stress path test of Toyoura sand by Pradhan et al. [14]. The sand contains granular quartz crystal particles. The initial void ratio is 0.845 , the initial spherical stress is $98.1 \mathrm{kPa}$, the normal compression line intercept is $N=0.969$, and the initial over consolidation stress ratio is $R=1.0$. The material parameters are shown in Table 4.

Figure $6 \mathrm{a}$ is the relation curve between deviatoric strain and stress ratio. The figure shows that the predicted value is in good agreement with the experimental value in the triaxial compression, but the predicted value is larger than the experimental value when the state is being in triaxial extension. Figure $6 \mathrm{~b}$ is the relation curve between deviatoric strain and volume strain. It can be seen from the figure that it is in good agreement with the 
$\mathrm{T}$ a b 1 e 4

Material Parameters for Toyoura Sand

\begin{tabular}{||c|c|c|c|c|c|c|c|c||}
\hline$M$ & $c_{t}$ & $c_{e}$ & $v$ & $b_{r}$ & $m_{b}$ & $\alpha$ & $m$ & $\alpha_{c}$ \\
\hline 1.24 & 0.0030 & 0.0010 & 0.3 & 1.5 & 0.6 & 0.2 & 0.5 & 0.0118 \\
\hline
\end{tabular}

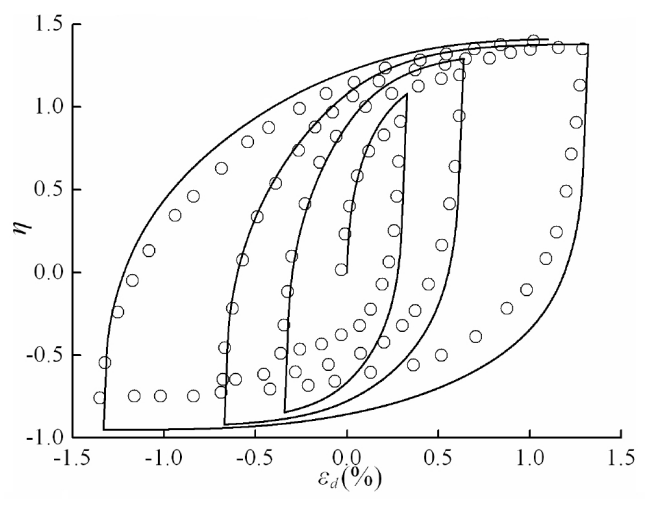

a

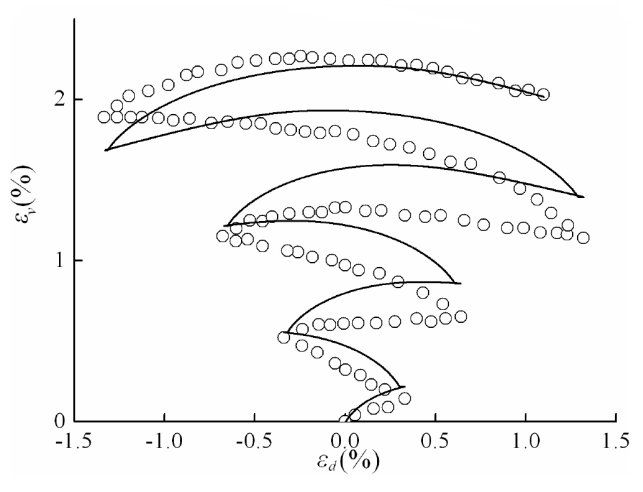

b

Fig. 6. Test and simulated results with a constant mean stress $p=98.1 \mathrm{kPa}$ on loose Toyoura sands.

experimental value, and the cyclic accumulation characteristics of the volume deformation can be fully reflected.

Figure 7 is a dense Toyoura sand of the same material. Figure $7 \mathrm{a}$ is the relationship curve of the deviatoric strain and stress ratio for dense sand, the initial void ratio is 0.653 , and the initial excess consolidation ratio is $R=0.005$. It can be seen from the diagram that when the sand becomes dense, the peak stress of sand is much higher than that of loose sand, but the shear strain amplitude does not decrease obviously, while the deviatoric strain predicted by the model is smaller than the experimental value. Figure $7 \mathrm{~b}$ is the corresponding relationship curve of stress ratio and volume strain, it can be seen that in the triaxial compression, compression volume strain predicted is consistent with that of test data, but the volume strain predicted of triaxial extension is larger. However, the predicted values of dilatancy are smaller than the experimental values, regardless of the triaxial compression or the triaxial extension.

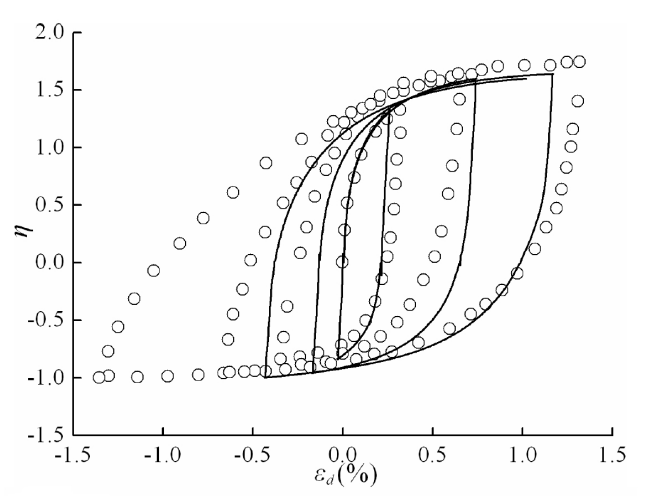

a

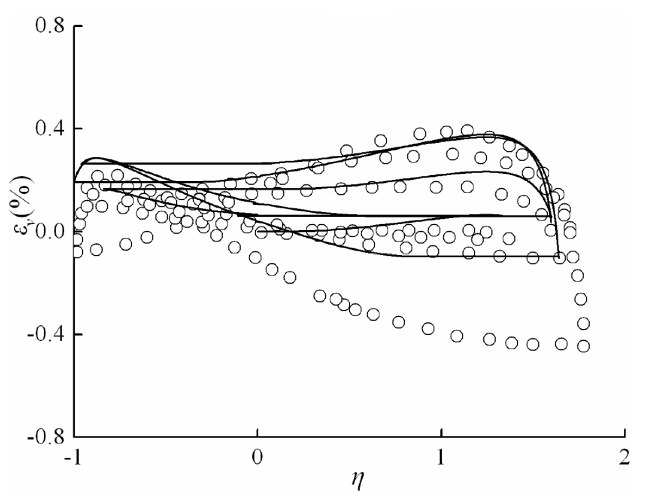

b

Fig. 7. Test and simulated results with a constant mean stress $p=98.1 \mathrm{kPa}$ on dense Toyoura sands. 
The peak strength of triaxial compression predicted in Fig. 7a is smaller than that of the experimental value, while the strength of triaxial extension is consistent with that of experimental value. The lower potential strength leads to the smaller volume strain of dilatancy.

Conclusions. By unified hardening over consolidation soil model, using the rotational hardening rule, the normal compression line and critical state line were modified, and the modification makes the model better to simulate the stress-strain relationship of saturated sand. The model requires less material parameters, namely nine parameters with a clear physical meaning, which can be determined by conventional basic experiments. Under a broad range of confining pressure, the coarse-grained soil and sand can be better simulated. The stress paths and the sand with different materials and states are simulated, and the simulation results show the rationality of the proposed model.

Acknowledgments. This study was supported by the National Natural Science Foundation of China for young scholars (Grant No. 11402260).

1. R. Verdugo and K. Ishihara, "The steady state of sandy soils," Soils Found., 36, No. 4, 81-91 (1996).

2. J. A. Yamamuro and P. V. Lade, "Drained sand behavior in axisymmetric tests at high pressures," J. Geotech. Eng.-ASCE, 122, No. 2, 109-119 (1996).

3. D. Wood, Soil Behaviour and Critical State Soil Mechanics, Cambridge University Press, Cambridge (1991), DOI: 10.1017/CBO9781139878272.

4. K. Hashiguchi, "On the linear relations of $V-\ln p$ and $\ln V-\ln p$ for isotropic consolidation of soils," Int. J. Numer. Anal. Met., 19, No. 10, 367-376 (1995).

5. T. Nakai, "An isotropic hardening elastoplastic model considering the stress path dependency in three-dimensional stresses," Soils Found., 29, No. 1, 119-139 (1989).

6. G. Gudehus, "A comprehensive constitutive equation for granular materials," Soils Found., 36, No. 1, 1-12 (1996).

7. K. Ishihara and S. Yasuda, "Sand liquefaction due to irregular excitation," Soils Found., 12, No. 4, 65-77 (1972).

8. J. M. Zhang, Y. Shamoto, and K. Tokimatsu, "Moving critical and phase transformation stress state lines of saturated sand during undrained cyclic shear," Soils Found., 37, No. 2, 1-59 (1997).

9. X. S. Li and Y. Wang, "Linear representation of steady-state line for sand," $J$. Geotech. Geoenviron., 124, No. 12, 1215-1217 (1998).

10. Y. P. Yao, D. C. Lu, A. N. Zhou, and B. Zou, "Generalized non-linear strength theory and transformed stress space," Sci. China Ser. E-Technol. Sci., 47, No. 6, 691-709 (2004).

11. D. A. Sun, W. X. Huang, D. C. Sheng, and H. Yamamoto, "An elastoplastic model for granular materials exhibiting particle crushing," Key Eng. Mat., 341, 1273-1278 (2007).

12. A. S. Saada, P. Puccini, and G. Bianchini, "Information package," in: A. S. Saada and G. Bianchini (Eds.), Constitutive Equations for Granular Non-Cohesive Soils, Balkema, Rotterdam (1989), pp. 81-88.

13. K. Ishihara, F. Tatsuoka, and S. Yasuda, "Undrained deformation and liquefaction of sand under cyclic stresses," Soils Found., 15, No. 1, 29-44 (1975).

14. T. B. S. Pradhan, F. Tatsouka, and Y. Sato, "Experimental stress-dilatancy relations of sand subjected to cyclic loading," Soils Found., 29, No. 1, 45-64 (1989).

Received 05. 03. 2018 